\title{
Rare Tumours of the Testis: Twelve Years of Experience
}

\author{
Zeynep Oruc, Senar Ebinç, M. Ali Kaplan \\ Department of Medical Oncology, Faculty of Medicine, Dicle University, \\ Diyarbakır, Turkey
}

Received May 21, 2020; Accepted September 14, 2020.

Key words: Testis tumours - Rare tumours - Gonadal tumours

Abstract: Rare tumours of the testis includes a wide variety of tumours. We aim to present clinical and histological characteristics of our patients with rare tumours of the testis. The medical records of 33 patients who were treated and followed-up for testicular rare tumours in our center between 2007 and 2020 were retrospectively reviewed. Of all the 243 testicular tumours, 222 cases (91.4\%) were germ cell tumours and 21 cases (8.6\%) were non-germ cell tumours. Thirty-three rare tumours of the testis including rare germ cell tumours and nongerm cell tumours were detected. The mean age of the patients at diagnosis was 34 years (range 18-68 years). The histological types of rare testicular tumours were as follows: teratoma $4.5 \%(n=11)$, sex-cord stromal tumours $4.5 \%(n=11)$, paratesticular tumours 3.2\% $(n=8)$, and the others [lymphoma $0.4 \%(n=1)$, mesothelioma $0.4 \%(n=1)$ and choriocarcinoma $0.4 \%(n=1)]$. The median duration of follow-up was 32 months (range 1 to 256 months). None of the patients with non-metastatic disease stage developed recurrence after having received appropriate therapy. Metastatic disease was documented in 9 cases at the time of diagnosis (five patients with teratomas, two patients with Leydig cell tumour, one patient with choriocarcinoma and rhabdomyosarcoma). The most common subtypes of testicular rare tumours in our center was teratoma and sex-cord stromal tumours. Because of testicular rare tumours have different biological features and different clinical outcomes, the management of each tumour requires a different approach.

Mailing Address: Zeynep Oruc, MD., Department of Medical Oncology, Faculty of Medicine, Dicle University, Billstreet. Sur, 20280 Diyarbakır, Turkey; Mobile Phone: 050637148 96; e-mail: zeynep44oruc@hotmail.com 


\section{Introduction}

Testicular tumours constitute less than $1 \%$ of all male cancers and occur especially between the ages of 15 and 35 years. Testicular germ cell tumour is the most common type of testicular malignancies (approximately $95 \%$ of all testicular tumours). Testicular germ cell tumours include a heterogeneous group of neoplasms such as seminoma, yolk sac tumours, embryonal carcinoma, mixed germ cell tumours, teratoma, and choriocarcinoma (Williamson et al., 2017). The remaining (5\%) involves non-germ cell tumours such as sex-cord stromal tumours, testicular lymphomas, and paratesticular tumours [sarcoma (liposarcoma, leiomyosarcoma, rhabdomyosarcoma)] (Williamson et al., 2017; Mooney and Kao, 2018).

The clinical features of more common germ cell tumours such as seminoma, yolk sac tumours, embryonal carcinoma, mixed germ cell tumours are well known. The optimal treatment approaches have been standardized, and this has led to excellent treatment outcomes (Howitt and Berney, 2015). However, we know little about rare testicular cancers including teratoma, choriocarcinoma, and non-germ cell tumours.

There are no studies involving large series of patients with rare tumours of the testis that would guide management, and available data is limited to the case reports and case series. Therefore, it is important that the centers share their own experiences.

The main objective of the present study was to analyse the cases documented in our department to determine the relative frequency of testicular rare tumours in adults and to report clinical and histological characteristics, treatment and outcomes of these tumours.

\section{Material and Methods}

We performed a retrospective analysis of patients with testicular tumours in the archived records between 2007 and 2020. Clinical characteristics, histopathological findings, laboratory values and treatment data of the patients were recorded from the hospital records and computer data system. A total of 243 cases with testicular tumour have been reviewed. All the cases of germ cell and non-germ cell tumours were separated according to the histological subtype. Also, the files of patients diagnosed with teratoma and choriocarcinoma, which are rare from germ cell tumours, were separated.

Thirty-three patients with rare tumours of the testis including rare germ cell tumours and non-germ cell tumours were identified (Table 1). The parameters evaluated were as follows: age at diagnosis, clinical characteristics (presentation at diagnosis), preoperative serum tumour marker [alpha fetoprotein (AFP), human chorionic gonadotropin (HCG)] levels, histopathological findings, stage, treatment methods, and treatment outcome.

\section{Results}

Of all the 243 testicular tumours, 222 cases $(91.4 \%)$ were germ cell tumours and 21 cases $(8.6 \%)$ were non-germ cell tumours. Of all germ-cell tumours, $36.6 \%(n=89)$ 


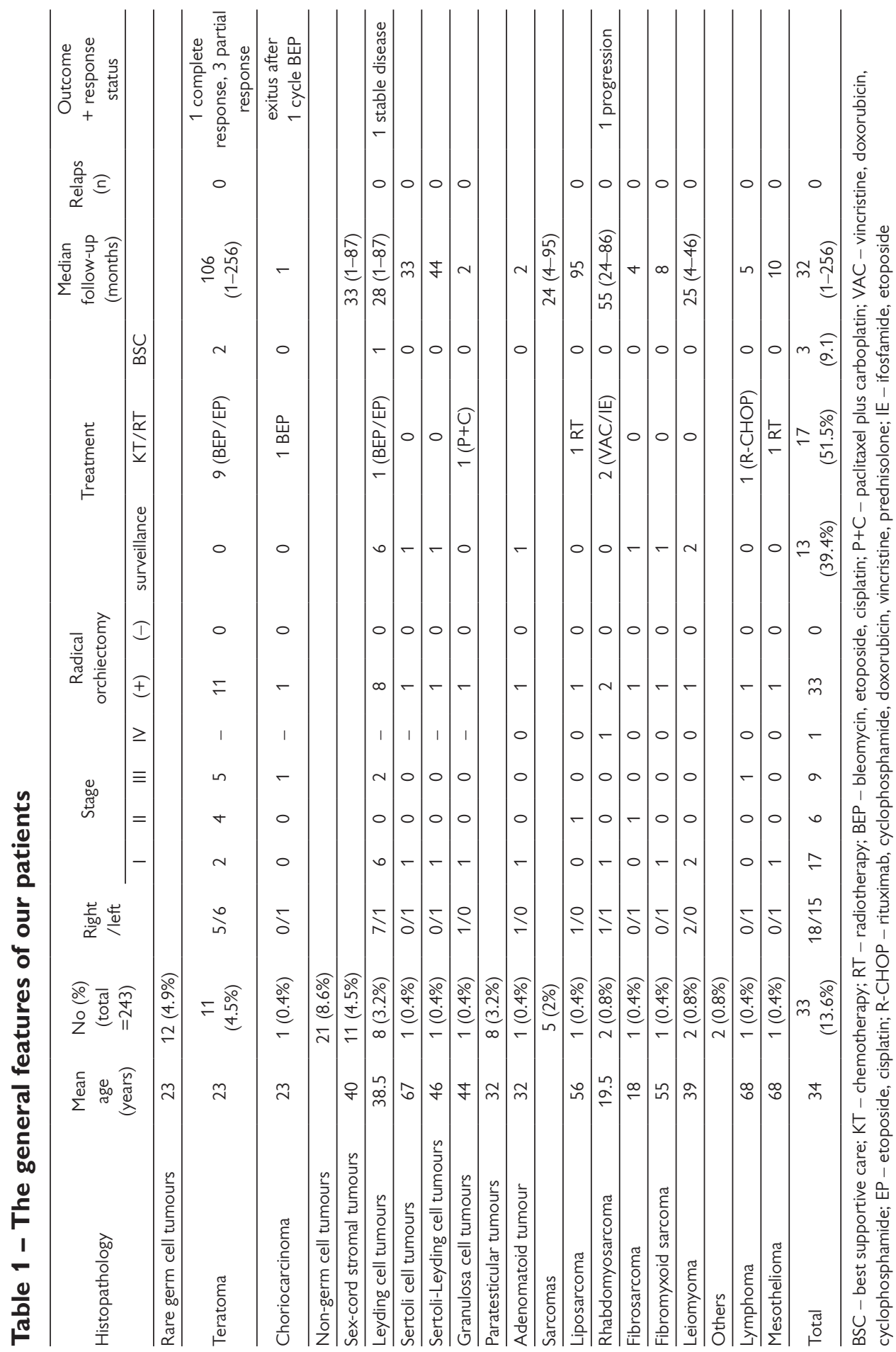




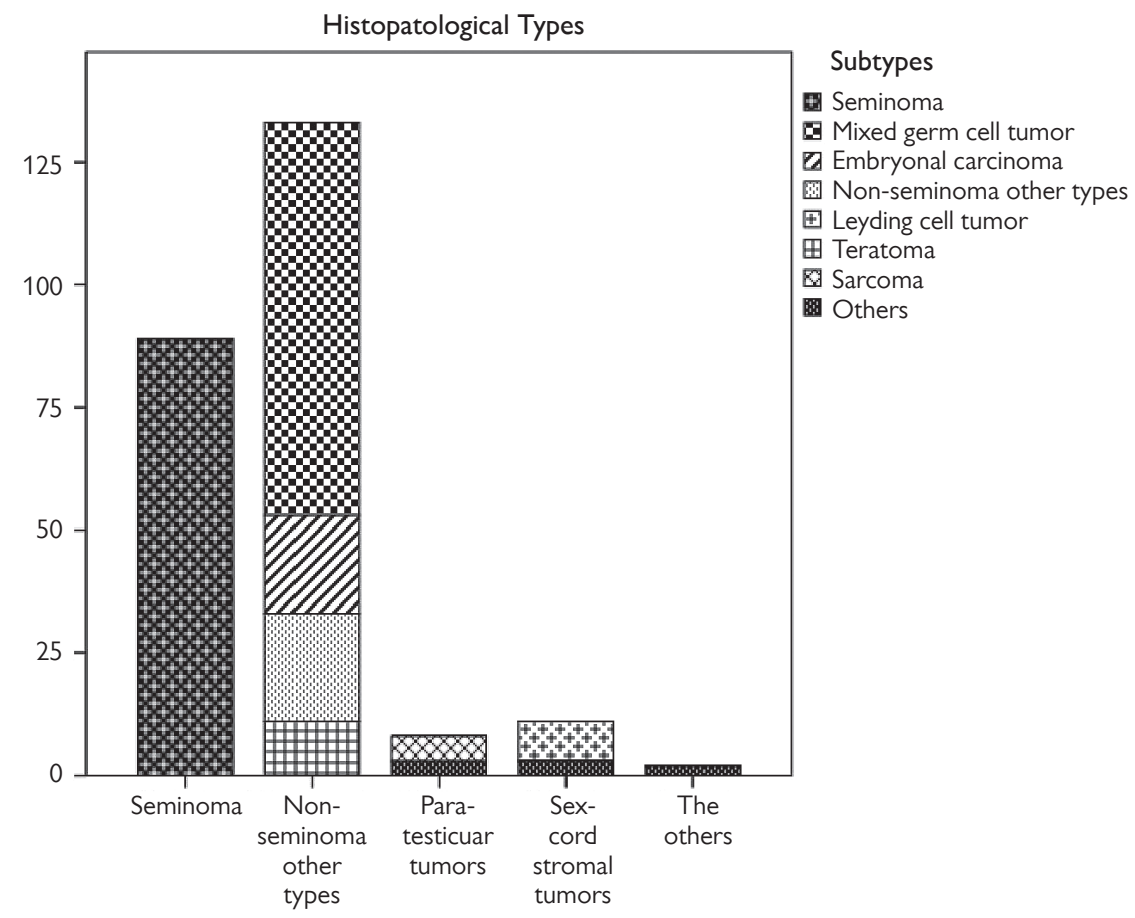

Figure 1 - Histopathological types and subtypes of testicular tumours.

were seminoma, 32.9\% $(n=80)$ were mixed germ cell tumour, $8.6 \%(n=21)$ were yolk sac tumour, 8.4\% $(n=20)$ were embryonal carcinoma, and 4.9\% $(n=12)$ were rare germ cell tumours (teratoma and choriocarcinoma).

The clinical data of the 33 patients who were diagnosed with rare tumour of the testis (non-germ cell tumours and rare germ cell tumours) are presented in Table 1. The mean age at presentation was 34 years (range 18-68 years). The most common presentation (>95\%) was a painless scrotal mass or swelling. Two patients had a history of trauma, and one of the patients had a history of cryptorchidism. No family history was reported. None of the patients had endocrine symptoms such as gynecomasty. All 33 testicular tumours were treated with radical orchiectomy. The median size of primary tumour mass in the testicle was $43 \mathrm{~mm}$ (range 5-170 mm). The diameters of most tumours were more than $4 \mathrm{~cm}$. The median duration of follow-up in all patients was 32 months (range 1-256 months). With regard to the laterality, the tumour was located in the right in 18 patients $(54.5 \%)$ and in the left in 15 patients $(45.5 \%)$.

The histological subtypes of rare testicular tumours were as follows: teratoma, 4.5\% $(n=11,11 / 243)$; sex-cord stromal tumours, 4.5\% $(n=11)$; paratesticular tumours, 3.2\% $(n=8)$; and others [lymphoma 0.4\% $(n=1)$, mesothelioma $0.4 \%(n=1)$ 
and choriocarcinoma $0.4 \%(n=1)]$. The rates of histopathological subtypes are shown in detail in Figure 1.

The mean age of the patients with testicular neoplasms of different histology were 23, 40 and 32 years in patients with teratoma, sex-cord stromal tumours and paratesticular tumours, respectively. In histopathological examination, the presence of necrosis, mitotic activity $(>5)$ and angiolymphatic invasion were observed in stage III Leydig cell tumours. Also, necrosis and mitotic activity $(>5)$ were reported in liposarcoma and rhabdomyosarcoma.

Based on our experience of more than 12 years on the rare tumours of the testis, we found that 11 cases (4.5\%) had sex-cord stromal tumours [eight cases with Leydig cell (six cases at stage I and 2 case at stage III (M1)], one case with Sertoli (at stage I), one case with Sertoli-Leydig cell (at stage I) and one case with granulosa (at stage I). The patient diagnosed with high risk stage I granulosa cell tumour underwent retroperitoneal lymph node dissection (RPLND) and received adjuvant carboplatin plus paclitaxel. Of these 2 patients, one underwent chemotherapy (BEP/EP) (BEP - bleomycin, etoposide, cisplatin) (EP - etoposide, cisplatin) and had stable disease at follow-up; the remaining one patient who did not receive chemotherapy and was followed with best supportive care, showed progression.

In total, 11 out of 33 (4.5\%) patients were diagnosed with teratoma. Testicular teratomas comprised $4.9 \%(11 / 222)$ of patients with germ cell tumours. Five patients with teratoma presented with stage III disease (2 cases had stage I and four cases had stage II disease) and 1 patient with choriocarcinoma had stage III disease (M1). One patient with testicular lymphoma presented with stage III disease. One patient with mesothelioma had stage I disease. Of patients with paratesticular tumours, two had rhabdomyosarcoma (one had stage I and the other had stage IV), two patients had leiomyoma, one patient had stage I fibromyxoid sarcoma, one patient had stage II liposarcoma, one patients had stage II fibrosarcoma, and one patient had adenomatoid tumour. Of the 33 patients, two patients had benign tumours, including one patient with leiomyoma $(0.8 \% ; 2 / 243)$ and one patient with adenomatoid tumour $(0.4 \%)$.

Serum $\beta$-HCG ( $\beta$-human chorionic gonadotropin) was elevated in three patients with stage III teratoma and one patient with choriocarcinoma. In addition, LDH (lactate dehydrogenase) was elevated in patients with stage III teratoma, lymphoma, stage IV rhabdomyosarcoma and chorciocarcinoma.

Adjuvant chemotherapy was administered to eight patients with early stage rare testicular tumour according to the histological subtype [BEP regimen in 6 patients diagnosed with teratoma, VAC/IE (VAC - vincristine, doxorubicin, cyclophosphamide; IE - ifosfamide, etoposide) regimen in 1 patient with rhabdomyosarcoma, carboplatin plus paclitaxel regimen in 1 patient with granulosa cell tumour], and 2 patients received only adjuvant radiotherapy ( 2 cases diagnosed with mesothelioma and liposarcoma). R-CHOP (rituximab, cyclophosphamide, doxorubicin, vincristine, prednisolone) was given to the patient with a testicular 
lymphoma. Thirteen patients were actively followed-up after radical orchiectomy since they had low-risk early stage disease. None of the patients with non-metastatic disease developed recurrence after having received appropriate therapy and being follow-up [median duration of follow-up, 32 months (range 1-256)] without evidence of disease.

Metastatic disease was documented in 9 of these cases at the time of diagnosis. Three out of nine patients with metastatic disease (two cases with teratoma, one case with Leydig cell tumour) received no palliative therapy and were followed-up with best supportive care because of poor performance status. The remaining six patients received chemotherapy pursuant to the histological subtype (BEP in patients with teratomas and choriocarcinoma, carboplatin and paclitaxel in patients with Leydig cell tumour, VAC/IE in patients with alveolar rhabdomyosarcoma). One patient with choriocarcinoma died of metastatic disease after the first cycle of the BEP regimen. Complete response was observed in one of the patients with a metastatic teratoma, and partial response was achieved in two patients.

A patient with rhabdomyosarcoma had progressive disease at the end of 3 months after having received the $\mathrm{VAC/IE}$ regimen. A patient with a metastatic Leydig cell tumour showed stable response to the BEP regimen, and the patient died of metastatic disease (median duration of follow-up was 28 months). Twenty-four patients survived (median duration of follow-up, 32 months), whereas 9 died of active disease. The deaths occurred in patients with Leydig cell tumour, teratoma, choriocarcinoma, rhabdomyosarcoma, mesothelioma and lymphoma.

\section{Discussion}

Rare tumours of the testis include a wide variety of tumours such as non-germ cell tumours and rare germ cell tumours (teratoma and choriocarcinoma), each presenting with different histopathological and clinical characteristics. Therefore, many different treatment approaches require for some histological types of rare testis tumours.

In general, a painless testicular mass or swelling, occasionally accompanied by a hydrocele is probably the typical presenting symptom of the rare tumours of the testis, as it is within in testicular germ cell tumours (Howitt and Berney, 2015). In our study, the most common presenting symptom (>95\%) was a painless scrotal mass or swelling. We also observed that there was a mild predominance of right-sided teratomas (right/left, 54.5\%/45.5\%). As in germ cell tumours, there is probably no clear evidence for a predisposition of one side due to limited data (Dieckmann et al., 2018).

Testis tumours are mainly observed between the ages of 20 and 45 years. However, rare tumours of the testis have a different age distribution. Malignant Leydig cell tumours, testicular lymphomas, and paratesticular sarcomas are rare testicular tumours observed in individuals older than 60 years of age (Gigantino et al., 2013). But in our study, the mean age at presentation was 34 years (range 
18-68 years). The mean age of the patients with testicular neoplasms of different histological subtypes was 23,40 and 32 years in the groups of teratoma, sex-cord stromal tumours and paratesticular tumours, respectively.

Serum AFP (alpha-fetoprotein) and $\beta$-HCG are important tumour markers in detecting primary, recurrent, or metastatic disease as well as in follow-up of germ cell tumours. Teratomas and choriocarcinoma are rare tumours of the testis that can lead to increased serum AFP or $\beta$-HCG concentrations. But tumour markers are negative in all sex-cord stromal tumours and thus routine measurement during follow-up is not required (Azizi et al., 2020). Three patients with metastatic teratomas and one patient with choriocarcinoma had elevated $\beta-H C G$ levels before and after surgery. In addition, LDH elevation was detected in all patients with stage III (M1) teratoma, lymphoma, stage IV rhabdomyosarcoma and choriocarcinoma. The remaining patients had normal serum markers.

In adult patients with testicular tumours, the specific histological diagnosis is based on the resected specimen. In daily practice, as malignant tumours of the testis are far more common than benign tumours in adulthood, radical orchiectomy has become standard approach among the urologic surgeons (Gigantino et al., 2013). In our study, of 243 patients with testicular tumours, 222 (91.4\%) had germ cell tumours and $21(8.6 \%)$ had non-germ cell tumours. Thirty-three patients with rare tumours of the testis including rare germ cell tumours and non-germ cell tumours were identified. We showed that in our center, most of the rare tumours of the testis were malignant. The most common histological subtype of the rare testicular tumours was teratoma (4.5\%) and sex-cord stromal tumours (4.5\%). Our results are consistent with the rate of testicular cancer types reported in previous studies (David et al., 2017; Osbun et al., 2017).

The 5-year survival rate of patients with testicular germ cell tumours is more than 90\% (Williamson et al., 2017). But 5-year survival rate for rare testicular tumours and the prognosis of patients with these tumours differ across the tumour subtypes. The incidence and frequency of each tumour subtype varies considerably. Also, therapeutic modalities including surgery, radiation, and chemotherapy varies according to the histological type and stage (Azizi et al., 2020).

Pure teratoma of the testis is uncommon in adults, accounting for fewer than $5 \%$ of all testicular germ cell tumours. Teratomas may contain mature or immature elements and present as a component of mixed testicular germ cell tumours or rarely present as pure neoplasms (Cheng et al., 2017). Also, testicular teratomas can occasionally show transformation into somatic-type malignancies (Mikuz and Colecchia, 2012). In the latest World Health Organization (WHO) classification, pure testicular teratoma is divided into two main forms based in part on the patient's age: prepubertal and postpubertal types. Postpubertal-type teratoma is clinically aggressive, while pure prepubertal-type teratoma has an indolent clinical course (Cheng et al., 2017). The standard treatments are radical surgery and cisplatin-based chemotherapy and/or radiotherapy based on tumour stage for germ 
cell tumours. Also, retroperitoneal lymphadenectomy is recommended in case of residual lesion after chemotherapy. In a retrospective study of 543 cases diagnosed with germ cell tumour, only 14 (2.5\%) had pure teratomas. Adult patients with malignant testicular teratomas consisted of $79 \%$ of all patients with pure teratoma cases in this series. Patients with testicular teratomas consisted of $4.9 \%(11 / 222)$ of patients with germ cell tumours in our study that involved 243 patients. Five cases with teratoma presented with advanced disease [stage III (M1)]. All of the cases had malignant teratoma. Three of the cases received curative BEP regimen. Complete response was achieved in one patient with metastatic teratoma, and partial response was achieved in two patients.

Sex-cord stromal tumours constitute the great majority of non-germ cell tumours of the testis, accounting for only $4 \%$ of all testicular tumours (Mooney and Kao, 2018). Leydig cell tumours and Sertoli cell tumours are the most common subtypes, accounting for $1-2 \%$ and $0.5 \%$ of all testis tumours, respectively (Conkey et al., 2005). Granulosa cell tumour is a rare type of sex-cord stromal tumours that are represented by juvenile and adult subtypes. Endocrine symptoms [gynecomastia (>15\%), rarely Cushing syndrome, precocious puberty] are frequently observed in Leydig and granulosa cell tumours. Germ cell tumours and sex-cord stromal tumours are seen more commonly in young patients and are mostly benign, and approximately $5 \%$ to $10 \%$ have malignant behaviour (Conkey et al., 2005; Dieckmann et al., 2019). Patients with stage I disease and malignant characteristics had a lower 5-year survival rate (77-94\%) compared to those with stage I germ cell tumour (98\%). Also, five-year survival of patients with stage I Sertoli cell tumours is significantly lower than in those with Leydig cell tumour. Patients with metastatic disease have poor survival and these tumours are usually refractory to chemotherapy and radiotherapy (Banerji et al., 2016).

There is a lack of consensus on the optimal treatment approach in cases with testicular rare tumours. Most information regarding treatment and survival outcomes of malignant sex-cord stromal tumours come from small retrospective studies. Radical orchiectomy alone is generally curative for early stage sex-cord stromal tumours and benign tumours. The other options include retroperitoneal lymph node dissection (RPLND), radiation, chemotherapy or a combination therapy (Banerji et al., 2016; Azizi et al., 2020). Chemotherapy regimens have included bleomycin, etoposide and cisplatin, or doxorubicin and cisplatin (platinum regimens). In the absence of metastatic disease on imaging studies, active surveillance may be also a reasonable option. Primary RPLND with or without adjuvant chemotherapy may be performed in selected patients with sex-cord stromal tumours, especially in highrisk patients. For patients with clinical stage I sex-cord stromal tumours with two or more malignant characteristics, primary RPLND is a recommended treatment strategy (Azizi et al., 2020).

In a study involving a total of 48 men with testicular sex-cord stromal tumours [Leydig cell (28), Sertoli cell (13), granulosa cell (2) and unclassified (5) tumours], 
Silberstein et al. (2014) reported 34 patients with no malignant characteristics at partial or radical orchiectomy who were successfully followed-up without primary RPLND and experienced no tumour recurrence during a limited follow-up duration (median 14.5 months). In our study, we found that 11 cases (4.5\%) had sex-cord stromal tumours [eight patients had Leydig cell (3.2\%), one patient had Sertoli $(0.4 \%)$, one patient had Sertoli-Leydig ( $0.4 \%)$, and one patient had granulosa $(0.4 \%)]$. None of the patients had a history of gynecomasty. Six patients presented with stage I Leydig tumour at the time of initial diagnosis, and two patients had stage III disease (M1). Of these 2 patients, one underwent chemotherapy (BEP) and had stable disease during the follow-up; the remaining patient who did not receive chemotherapy and was followed with best supportive care showed progression. Adjuvant therapy was not administered to six patients with stage I Leydig cell tumour and 3 patients with the other types of sex-cord stromal tumours and not directed to RPLND since they did not have malignant characteristics. These patients remained disease-free during a follow-up period of 33 months after radical orchiectomy. But a patient who was diagnosed with high-risk stage I granulosa cell tumour underwent RPLND and received adjuvant carboplatin plus paclitaxel. Poor prognostic characteristics such as large size ( $5 \mathrm{~cm}$ or greater), necrosis, nuclear atypia, vascular invasion, high mitotic rate (more than 5 per 10 high-power fields) and age $>50$ years increase the likelihood of malignancy in a testicular mass (Bremmer et al., 2014; Mooney and Kao, 2018).

Paratesticular tumours originate from the paratesticular soft tissue region (spermatic cord, epididymis and tunica vaginalis). These tumours are rare, accounting for fewer than $5 \%$ of all testicular masses. The majority of all paratesticular masses are benign and include lipomas, adenomatoid tumours, fibroma, hemangioma, and leiomyomas. Adenomatoid tumours are the most common paratesticular tumours occurring predominantly in patients aged 30 to 50 years as an asymptomatic and slow-growing mass. Surgical excision is curative because it is benign (Amin, 2005; Mooney and Kao, 2018). In our study, the benign tumours were leiomyoma and adenomatoid tumour. There were two patients with leiomyoma and one patient with adenomatoid tumour. The rate of benign lesions may be underestimated, because benign tumours are less likely to be referred to the department of oncology.

Paratesticular sarcomas occur rarely in adults, with a peak incidence between 55 and 65 years of age (Keenan et al., 2019). Approximately 30\% of paratesticular tumours have malignant histology. Malignant paratesticular sarcomas include liposarcomas, rhabdosarcomas, leiomyosarcomas and malignant fibrous histiocytomas (Keenan et al., 2019). Liposarcoma is the most common type of paratesticular sarcomas. The majority are well-differentiated sarcomas. Recurrence can occur locally in well-differentiated sarcomas but have no metastatic potential in the absence of dedifferentiation. Dedifferentiated sarcomas have the potential to metastasize (Mooney and Kao, 2018; Keenan et al., 2019). The main prognostic 
factors are tumour size, grade and nodal or distant metastasis (Gigantino et al., 2013). Overall five-year cancer-free survival ranges from approximately $60 \%$ to $80 \%$ (Khoubehi et al., 2002). In our study, paratesticular sarcomas were as follows; two cases with rhabdomyosarcoma at stage I and at stage IV $(0.8 \%)$, one case with fibromyxoid sarcoma at stage I $(0.4 \%)$, one case with liposarcoma at stage II $(0.4 \%)$, one fibrosarcoma at stage II ( $0.4 \%)$. Rhabdomyosarcoma is a rare tumour, mostly seen in the first two decades of life unlike the other paratesticular sarcomas. Rhabdomyosarcomas tend to be more aggressive than paratesticular tumours. Rhabdomyosarcoma was reported to have a high incidence of lymph nodes metastasis and distant metastasis.

There is no consensus on the optimal treatment approach in patients with paratesticular sarcoma. However, aggressive surgical resection should be performed in patients with paratesticular sarcomas to ensure surgical margin negativity (Keenan et al., 2019). The role of retroperitoneal lymph node dissection is uncertain, especially in liposarcoma and leiomyosarcoma. Retroperitoneal lymph node dissection is recommended in some cases of intermediate-grade or high-grade lymph node involvement on imaging studies of patients with liposarcoma and leiomyosarcoma, and in all patients over 10 years of age as part of staging for rhabdomyosarcomas (Khoubehi et al., 2002; Dangle et al., 2016). Some experts advocate the use of chemotherapy in metastatic disease. Adjuvant chemotherapy in paratesticular sarcomas has not been evaluated in previous studies. The local relapse rate after orchiectomy is $25-37 \%$ for paratesticular tumours. Adjuvant radiotherapy reduces locoregional recurrence (Khoubehi et al., 2002). Fagundes et al. (1996) have reported no local recurrence in patients receiving adjuvant radiation after orchiectomy, compared with those treated with orchiectomy alone (37\%). In our study, a patient diagnosed with stage II liposarcoma received only adjuvant radiotherapy. This patient is followed in recurrence-free remission.

The current treatment has been standardized for rhabdomyosarcoma unlike the other paratesticular sarcomas. Multimodal approach including radical orchidectomy and/or RPLND, multiagent chemotherapy (VAC/IE regimen) and/or radiation is required (Dangle et al., 2016). The patients with stage I rhabdomyosarcoma received VAC/IE regimen without RPLND. The patient at stage $I$ is followed free of recurrence. The patient at stage IV had progressive disease at the end of 3 months after $\mathrm{VAC} / \mathrm{IE}$ regimen and died of metastatic disease at twenty-four months of the disease.

In our study, each of the other cases, including those with choriocarcinoma, mesothelioma and lymphoma of rare germ cell tumours, make up $0.4 \%$ of the total number of cases. Choriocarcinoma of the testis is extremely rare in the testis but can be seen as a component of a mixed germ cell tumour. Most patients are between 10 and 30 years of age. Serum $\beta$-HCG levels are markedly elevated (Cheng et al., 2017). The prognosis of choriocarcinoma is poor without treatment. But there is a chance of cure with combination chemotherapy even in patients with a 
metastatic disease (Howitt and Berney, 2015). Our patient with choriocarcinoma died of metastatic disease after 1 cycle BEP regimen.

Malignant mesothelioma of the tunica vaginalis testis is an extremely rare tumour, representing $0.3 \%$ to $5 \%$ of all malignant mesotheliomas (Chekol and Sun, 2012). It occurs most commonly in men in the sixth or seventh decades of life. Exposure to asbestos is the only known risk factor (Amin, 2005). The prognosis for patients with malignant mesothelioma is poor, with a median survival of 23 months (Chekol and Sun, 2012). Aggressive therapy with radical orchiectomy and chemotherapy for advanced or recurrent disease remains the mainstay of therapy, usually with poor response. Our patient had asbestos exposure. After orchiectomy, the patient received adjuvant radiotherapy due to risk factors $(>5 \mathrm{~cm}$ tumour size, rete testis and lymphovascular invasion).

Testicular lymphomas are rare, accounting for approximately $1-2 \%$ of all nonHodgkin lymphomas and 5\% of testicular malignancies (Gigantino et al., 2013). It is the most common type of testicular cancer in men older than 60 years of age. The majority of testicular lymphomas (80-98\%) are histologically diffuse large B-cell lymphoma (Twa et al., 2018). The patients with testicular lymphomas have the heightened risk of central nervous system (CNS) relapse. The prognosis for this lymphomas is poor. In a review, overall survival at 5 and 10 years for stage I patients was 58 and 29\%, and for stage II patients, it was 46 and 29\%, respectively (Zucca et al., 2003). But patients with stage IV disease have a relapse rate greater than $90 \%$ and a 5-year survival of 20-25\% (Gigantino et al., 2013). The standard treatment for testicular DLBCL (diffuse large B-cell lymphoma) generally includes orchiectomy and anthracycline, rituximab containing chemotherapy regimens, with or without locoregional radiotherapy and prophylactic scrotal radiotherapy in addition to central nervous system-directed prophylaxis (Cheah et al., 2014). There was a 68-year-old patient with testicular lymphoma in our study. The histopatology was follicular lymphoma. The patient was found to have stage III disease on imaging studies. The patient started a R-CHOP chemotherapy regimen. After five months, follow-up data is not available. There are case reports in literature on follicular lymphoma of the testis.

The limitations of this study include small sample size and retrospective study design. Despite these limitations, we presented our own data on rare tumours of the testis.

In conclusion, the most common subtypes of testicular rare tumours in our center were teratoma and sex-cord stromal tumours. Testicular rare tumours in adults have various tumour types, because of rare occurrence and limited clinical data of these tumours, optimal treatment strategies are challenging. Because testicular rare tumours have different biological features and different clinical outcomes, the management of each tumour requires a different approach. Clinical experience in testicular rare tumours is based on a few small case series and predominantly on single case reports. Therefore, it is important to make treatment decisions of patients with testis rare tumours in tumour boards. 


\section{References}

Amin, M. B. (2005) Selected other problematic testicular and paratesticular lesions: Rete testis neoplasms and pseudotumors, mesothelial lesions and secondary tumors. Mod. Pathol. 18, S131-S145 (Suppl. 2).

Azizi, M., Aydin, A. M., Cheriyan, S. K., Peyton, C. C., Montanarella, M., Gilbert, S. M., Sexton, W. J. (2020) Therapeutic strategies for uncommon testis cancer histologies: Teratoma with malignant transformation and malignant testicular sex cord stromal tumors. Transl. Androl. Urol. 9, S91-S103 (Suppl. 1).

Banerji, J. S., Odem-Davis, K., Wolff, E. M., Nichols, C. R., Porter, C. R. (2016) Patterns of care and survival outcomes for malignant sex cord stromal testicular cancer: Results from the National Cancer Data Base. J. Urol. 196, 1117-1122.

Bremmer, F., Behnes, C. L., Radzun, H. J., Bettstetter, M., Schweyer, S. (2014) Sex cord gonadal stromal tumors. Pathologe 35, 245-251.

Cheah, C. Y., Wirth, A., Seymour, J. F. (2014) Primary testicular lymphoma. Blood 123(4), 486-493.

Chekol, S. S., Sun, C. C. (2012) Malignant mesothelioma of the tunica vaginalis testis: Diagnostic studies and differential diagnosis. Arch. Pathol. Lab. Med. 136(1), 113-117.

Cheng, L., Lyu, B., Roth, L. M. (2017) Perspectives on testicular germ cell neoplasms. Hum. Pathol. 59, 10-25.

Conkey, D., Howard, G. C. W., Grigor, K. M., McLaren, D. B., Kerr, G. R. (2005) Testicular sex cord-stromal tumours: The Edinburgh experience 1988-2002, and a review of the literature. Clin. Oncol. 17(5), 322-327.

Dangle, P. P., Correa, A., Tennyson, L., Gayed, B., Reyes-Múgica, M., Ost, M. (2016) Current management of paratesticular rhabdomyosarcoma. Urol. Oncol. 34(2), 84-92.

David, S., Andras, F., Endre, K., Balint, K., Arpad, K., Csaba, P., Karoly, S., Tamás, T. (2017) More cases of benign testicular teratomas are detected in adults than in children. A clinicopathological study of 543 testicular germ cell tumor cases. Pathol. Oncol. Res. 23(3), 513-517.

Dieckmann, K. P., Richter-Simonsen, H., Kulejewski, M., Ikogho, R., Zecha, H., Anheuser, P., Pichlmeier, U., Isbarn, H. (2018) Testicular germ-cell tumours: A descriptive analysis of clinical characteristics at first presentation. Urol. Int. 100(4), 409-419.

Dieckmann, K. P., Bertolini, J., Wülfing, C. (2019) Adult granulosa cell tumor of the testis: A case report with a review of the literature. Case Rep. Urol. 2019, 7156154.

Fagundes, M. A., Zietman, A. L., Althausen, A. F., Coen, J. J., Shipley, W. U. (1996) The management of spermatic cord sarcoma. Cancer 77, 1873-1876.

Gigantino, V., La Mantia, E., Franco, R., Cecere, S., Rossetti, S., Napoli, M. D., Pisano, C., Berretta, M., Galzerano, A., Botti, G., Pignata, S., Facchini, G. (2013) Testicular and testicular adnexa tumors in the elderly. Anticancer Drugs 24(3), 228-236.

Howitt, B. E., Berney, D. M. (2015) Tumors of the testis: Morphologic features and molecular alterations. Surg. Pathol. Clin. 8(4), 687-716.

Keenan, R. A., Nic An Riogh, A. U., Stroiescu, A., Fuentes, A., Heneghan, J., Cullen, I. M., Daly, P. J. (2019) Paratesticular sarcomas: A case series and literature review. Ther. Adv. Urol. 11, 1756287218818029.

Khoubehi, B., Mishra, V., Ali, M., Motiwala, H., Karim, O. (2002) Adult paratesticular tumours. BJU Int. 90, 707-715.

Mikuz, G., Colecchia, M. (2012) Teratoma with somatic-type malignant components of the testis. A review and an update. Virchows Arch. 461(1), 27-32.

Mooney, K. L., Kao, C. S. (2018) A comptemporary review of common adult non-germ cell tumors of the testis and paratestis. Surg. Pathol. Clin. 11(4), 739-758.

Osbun, N., Winters, B., Holt, S. K., Schade, G. R., Lin, D. W., Wright, J. L. (2017) Characteristics of patients with Sertoli and Leydig cell testis neoplasms from a national population-based registry. Clin. Genitourin. Cancer 15(2), e263-e266.

Oruc Z.; Ebinç S.; Kaplan M. A. 
Silberstein, J. L., Bazzi, W. M., Vertosick, E., Carver, B. S., Bosl, G. J., Feldman, D. R., Bajorin, D. F., Motzer, R. J., Al-Ahmadie, H., Reuter, V. E., Sheinfeld, J. (2014) Clinical outcomes of local and metastatic testicular sex cord-stromal tumors. J. Urol. 192, 415-419.

Twa, D. D. W., Mottok, A., Savage, K. J., Steidl, C. (2018) The pathobiology of primary testicular diffuse large B cell lymphoma: Implications for novel therapies. Blood Rev. 32(3), 249-255.

Williamson, S. R., Delahunt, B., Magi-Galluzzi, C., Algaba, F., Egevad, L., Ulbright, T. M., Tickoo, S. K., Srigley, J. R., Epstein, J. I., Berney, D. M. (2017) The World Health Organization 2016 classification of testicular germ cell tumours: A review and update from the International Society of Urological Pathology Testis Consultation Panel. Histopathology 70(3), 335-346.

Zucca, E., Conconi, A., Mughal, T. I., Sarris, A. H., Seymour, J. F., Vitolo, U., Klasa, R., Ozsahin, M., Mead, G. M., Gianni, M. A., Cortelazzo, S., Ferreri, A. J., Ambrosetti, A., Martelli, M., Thiéblemont, C., Moreno, H. G., Pinotti, G., Martinelli, G., Mozzana, R., Grisanti, S., Provencio, M., Balzarotti, M., Laveder, F., Oltean, G., Callea, V., Roy, P., Cavalli, F., Gospodarowicz, M. K. (2003) Patterns of outcome and prognostic factors in primary large-cell lymphoma of the testis in a survey by the International Extranodal Lymphoma Study Group. J. Clin. Oncol. 21, 20-27. 